\title{
Existence of Solitary Waves in Higher Dimensions *
}

\author{
Walter A. Strauss
}

Department of Mathematics, Brown University, Providence, Rhode Island 02912, USA

\begin{abstract}
The elliptic equation $\Delta u=F(u)$ possesses non-trivial solutions in $\boldsymbol{R}^{\boldsymbol{n}}$ which are exponentially small at infinity, for a large class of functions $F$. Each of them provides a solitary wave of the nonlinear Klein-Gordon equation.
\end{abstract}

\section{Introduction}

We define a solitary wave as a solution $\phi(x, t)$ of a wave equation whose maximum amplitude at time $t, \sup _{x}|\phi(x, t)|$, does not tend to zero as $t \rightarrow \infty$, but which tends to zero in some convenient sense as $|x| \rightarrow \infty$ for each $t$. The convergence should have the property that physical quantities, such as the energy and charge, are finite. Particular types of solitary waves are (1) traveling waves $\phi=u(x-c t)$ where $c$ is a constant vector and (2) standing waves $\phi=\exp (i \omega t) u(x)$ where $\omega$ is a real constant. Traditionally, solitary waves have been traveling waves, but in recent years oscillatory factors have been allowed. The above definition includes all uses of the term. Solitary waves have also been called "solitons" but, properly speaking, the latter word should be reserved for those special solitary waves which exactly preserve their shapes after interaction. Many examples of these special solitons have been discovered in recent years in the case of two space-time dimensions. In higher dimensions, however, even the existence of solitary waves seems to be elusive.

We consider the scalar NLKG equation

$$
\phi_{t t}-\Delta \phi+m^{2} \phi+f(\phi)=0,
$$

where $x=\left(x_{1}, \ldots, x_{n}\right) \in \boldsymbol{R}^{n}, \Delta$ is the Laplacian in $x$ and $m>0$. We assume $f(0)=0$ and $f\left(r e^{i \theta}\right)=f(r) e^{i \theta}$. If $\phi$ is a standing wave (2), Equation (3) reduces to

$$
-\Delta u+\left(m^{2}-\omega^{2}\right) u+f(u)=0 \text {. }
$$

We shall show that (4) possesses non-trivial solutions exponentially small at infinity provided $|\omega|<m$ and $f$ satisfies certain conditions. In particular, if $\omega=0$, we have $\phi(x)=u(x)$. Since we may change to a different Lorentz frame, it follows that there exist traveling solitary waves $(1)$ for any $|c|<1$. Alternatively we may proceed by

^ This work was supported in part by NSF Grant MCS 75-08827 
putting (1) directly into (3) to obtain the equation

$$
-\sum a_{i j} \frac{\partial^{2} u}{\partial x_{i} \partial x_{j}}+m^{2} u+f(u)=0,
$$

where $a_{i j}=\delta_{i j}+c_{i} c_{j}$. Note that $\left(a_{i j}\right)$ is a positive-definite matrix because

$$
\sum a_{i j} \xi_{i} \xi_{j}=|\xi|^{2}-(c \cdot \xi)^{2} \geqq\left(1-|c|^{2}\right)|\xi|^{2}
$$

for any $\xi=\left(\xi_{1}, \ldots, \xi_{n}\right) \in \boldsymbol{R}^{n}$. Thus Equation (5) is elliptic and it can be converted into an equation with leading term $-\Delta v$ by means of a rotation and stretching of axes. The same sort of equation comes from standing wave solutions (2) of the NLS equation $i \phi_{t}-\Delta \phi+f(\phi)=0$.

In each of these cases we get an equation of the form

$$
-\Delta u+F(u)=0, \quad x \in \boldsymbol{R}^{n},
$$

where $F(u)=f(u)+$ (const.) $u$.

We assume that $F(0)=0$, which means that (6) always possesses the trivial solution $u=0$. Let $F$ be a real continuous function and let $G^{\prime}=F, G(0)=0$. Consider real-valued solutions of (6); for the complex case, see Section 2.

Some necessary conditions on $F$, found in part by Pohozaev [7], are the following.

Theorem 1. If $u$ is a solution of (6) which is sufficiently small at infinity, then

$$
(n-2) \int|\nabla u|^{2} d x=-(n-2) \int u F(u) d x=-2 n \int G(u) d x \text {. }
$$

Hence if $s F(s)$ or $G(s)($ for $n \neq 1)$ or $H(s)=(n-2) s F(s)-2 n G(s)$ or $-H(s)$ is positive (for $s \neq 0$ ), then the only solution is the trivial one. For any non-trivial solution, the energy is positive:

$$
E=\int\left[\frac{1}{2}|\nabla u|^{2}+G(u)\right] d x=\frac{1}{n} \int|\nabla u|^{2} d x>0 .
$$

The main result of this paper is the existence of non-trivial solutions of (6) for a large class of functions $F$. Let

$$
L=-\sum a_{i j} \frac{\partial^{2}}{\partial x_{i} \partial x_{j}}+a_{0},
$$

where the constant matrix $\left(a_{i j}\right)$ is positive-definite and $a_{0}$ is a positive constant. Let $F_{1}(s)$ and $F_{2}(s)$ be real continuous functions defined for $0 \leqq s<\infty$. Denote by $G_{1}$ and $G_{2}$ their indefinite integrals. Assume the following.

$$
F_{1}(s) \geqq 0, \quad F_{2}(s)>0 \quad \text { for } \quad s>0 .
$$

As $s \rightarrow 0, \quad F_{1}(s)=O(s) \quad$ and $\quad F_{2}(s)=o(s)$.

$F_{2}(s)=o\left(s^{l}+F_{1}(s)\right) \quad$ as $\quad s \rightarrow \infty$.

$$
F_{2}(s)=O\left(s^{l}+G_{1}(s) / s\right) \text { as } s \rightarrow \infty,
$$

where $l=(n+2) /(n-2)$ and $n \geqq 3$. (For the cases $n=1$ or 2 , see Section 3.)

Theorem 2. Under these assumptions, there exists $\lambda>0$ and a solution $u$ of

$$
L u+F_{1}(u)=\lambda F_{2}(u)
$$

which is non-negative, belongs to the Sobolev space $H^{1}$, decays exponentially as $|x| \rightarrow \infty$, and $\int G_{1}(u(x)) d x<\infty$. 
Examples are presented in Section 2.

A number of studies have been made of Equation (6) by restricting attention to the radial solutions $(u=u(r), r=|x|)$ and analyzing the resulting ordinary differential equation; see Synge [14], Nehari [6], Anderson and Derrick [1], and Berger [2]. One-dimensional problems have also been studied by Stuart [13] and Dancer [4]. In addition, there are a large number of papers on the existence of a solution of an equation with an inhomogeneous term $g(x)$; in the present case, when $g=0$, there is always the trivial solution $u=0$.

Nonlinear eigenvalue problems of this type have also been much studied for the case of a bounded domain $\Omega \subset \boldsymbol{R}^{n}$ with Dirichlet boundary conditions. Methods of the calculus of variations, bifurcation theory and topological degree have been used; see Pohozaev [7], Rabinowitz [9, 10], for example. These methods fail to extend to unbounded $\Omega$ because $H^{1}$ is no longer compact in $L^{2}$. To be compact in $L^{2}$ on an unbounded domain, a class of functions has to be uniformly small at infinity. We use the variational method with the extra constraint that the functions be radial, which ensures the compactness.

We allow rapid growth of the nonlinearities in Theorem 2. Almost all authors (for $\Omega$ bounded or not) require $F_{i}(s)=o\left(s^{l}\right)$ as $s \rightarrow \infty, i=1,2$. Clément [3], for the case of (12) in a bounded domain, relaxes this condition for $F_{1}$ but requires it for $F_{2}$. Rabinowitz [11] exhibits an example where $F_{2}$ does not satisfy the growth condition and $F_{1}=0$.

In Section 3 we prove Theorem 2 in two stages. We apply the direct variational method to truncated versions of $F_{1}$ and $F_{2}$. Then we remove the truncations by a limiting process, using techniques of Strauss [12] and Clément [3].

Classical eigenvalue problems possess an infinite sequence of solutions. By the minimax technique, the same should be true in our case. We have not carried this out; instead, in Section 4, we apply abstract theorems of Rabinowitz [10]. These theorems unfortunately require the growth conditions on $F_{1}$ and $F_{2}$ mentioned above.

I wish to thank L. Vazquez for many stimulatıng discussions on this topic and P. Rabinowitz and C. Dafermos for some helpful suggestions.

\section{Examples and Necessary Conditions}

We use the notation $L^{p}=L^{p}\left(\boldsymbol{R}^{n}\right),\|\|_{p}=L^{p}$-norm, $H^{1}=H^{1}\left(\boldsymbol{R}^{n}\right)=\left\{u \in L^{2} \mid \partial u / \partial x_{i} \in L^{2}\right.$, $i=1, \ldots, n\}$. Integrals are taken over all of $\boldsymbol{R}^{n}$ unless otherwise specified.

Example 1 (Pohozaev [7]).

$$
-\Delta u+u-|u|^{q-1} u=0 \text {, }
$$

$x \in \boldsymbol{R}^{n}, n \geqq 3, q>1$. We have scaled out the coefficients to be 1 . To apply Theorem 1 , $F(s)=s-|s|^{q-1} s, G(s)=s^{2} / 2-|s|^{q+1} /(q+1)$. Let $\alpha^{-1}=2^{-1}-(q+1)^{-1}$. So there is no non-trivial solution if $((n-2) / 2) s F(s)-n G(s)=-s^{2}+\left(1-\alpha^{-1} n\right)|s|^{q+1}$ is of one sign; that is, $\alpha \leqq n$ or $q \geqq(n+2) /(n-2)$. So assume $1<q<(n+2) /(n-2)$. Any solution must satisfy identity (7), which reduces to:

$$
\alpha(n-2) \int|\nabla u|^{2} d x=\frac{n \alpha}{\alpha-n} \int|u|^{2} d x=n \int|u|^{q+1} d x .
$$


Theorem 2 asserts the existence of a solution, where $F_{1}(s)=0, F_{2}(s)=|s|^{q-1} s$, $L=-\Delta+I$ and a scale change is used to make $\lambda=1$. In Section 4 we show that this solution is the first one of an infinite sequence of distinct solutions.

Example 2. $-\Delta u+\left(m^{2}-\omega^{2}\right) u+|u|^{p-1} u-\lambda|u|^{q-1} u=0$ where $x \in \boldsymbol{R}^{n}, m^{2}-\omega^{2}>0$ and $p$ and $q$ are distinct numbers larger than 1 . We distinguish four cases.

Case A: $q<p$. Theorem 2 asserts the existence of a non-trivial solution for some $\lambda>0$. Note that

$$
G(s)=\frac{1}{2}\left(m^{2}-\omega^{2}\right) s^{2}+\frac{1}{p+1}|s|^{p+1}-\frac{\lambda}{q+1}|s|^{q+1}
$$

is bounded below. There is a number $\lambda_{*}$ so that, for $\lambda \leqq \lambda_{*}, G(s)$ is non-negative and the only solution is the trivial one, according to Theorem 1.

According to Theorem 1, the (integrated) energy is necessarily positive, even though the function $G(s)$ is not allowed to be positive if a non-trivial solution is to exist. On the other hand, for a standing wave solution (2) of the NLKG Equation (3), the energy density

$$
\frac{1}{2}\left|\phi_{t}\right|^{2}+\frac{1}{2}|\nabla \phi|^{2}+G(\phi)=\frac{1}{2}|\nabla u|^{2}+\omega^{2} u^{2}+G(u)
$$

may be positive. This is the case if $\omega>0$ and $\lambda$ is slightly larger than $\lambda_{*}$.

Anderson [1] has computed these solutions in the case $n=3, p=5, q=3$. His most interesting result is that the positive solution appears to be stable with respect to perturbations of the initial data of Equation (3) in the cases when the energy density is positive. For this choice of $p, q$, and $n$, the inequalities

$$
\begin{aligned}
& \lambda s^{4}=(2 s)\left(\frac{1}{2} \lambda s^{3}\right) \leqq \frac{1}{2}(2 s)^{2}+\frac{1}{2}\left(\frac{1}{2} \lambda s^{3}\right)^{2}=2 s^{2}+\frac{1}{8} \lambda^{2} s^{6}, \\
& G(s)=\frac{1}{2} s^{2}+\frac{1}{6} s^{6}-\frac{\lambda}{4} s^{4} \geqq\left(\frac{1}{6}-\frac{1}{32} \lambda^{2}\right) s^{6}
\end{aligned}
$$

show that $\lambda_{*}=4 \cdot 3^{-1 / 2}$, if we normalize to make $m^{2}-\omega^{2}=1$. This explains the rather mysterious fraction $3 / 16=\lambda_{*}^{-2}$ appearing in [1].

Case B: $p<q<(n+2) /(n-2)$. Theorem 2 is again applicable. In Section 4 we prove the existence of an infinite sequence of non-trivial solutions for each $\lambda>0$.

Case $\mathrm{C}: p \leqq(n+2) /(n-2) \leqq q$. Let $\alpha^{-1}=2^{-1}+(q+1)^{-1}$ and $\beta^{-1}=2^{-1}$ $+(p+1)^{-1}$. Then $\alpha \leqq n \leqq \beta$ and

$$
\frac{n-2}{2} s F(s)-n G(s)=-s^{2}-\left(1-\frac{n}{\beta}\right)|s|^{p+1}+\left(1-\frac{n}{\alpha}\right) \lambda|s|^{q+1} \text {. }
$$

By Theorem 1, there can be no non-trivial solution.

Case $\mathrm{D}:(n+2) /(n-2)<p<q$. This case remains open: we do not know whether or not there exists a non-trivial solution.

Remark. For the equation $-\Delta u+F(u)=0$, existence essentially requires $F^{\prime}(0) \geqq 0$. Indeed, suppose $-\alpha=F^{\prime}(0)<0$ and let $f(s)=F(s) / s+\alpha$. Let $u(x)$ be a non-trivial 
solution small at infinity and let $q(x)=f(u(x))$. Then the equation can be rewritten as $-\Delta u+q u=\alpha u$. Assuming $u(x)$ is small enough at infinity that $q(x)=o\left(|x|^{-1}\right)$, it is well-known that the operator $-\Delta+q$ has no positive eigenvalues (Kato [5]), so that we reach a contradiction.

Remark. A word about real vs. complex solutions. If we restrict our consideration to real solutions $u(x)$, then we consider real functions $F(s)$ and $G(s)=\int_{0}^{s} F(\sigma) d \sigma$. If we allow $u(x)$ to be complex-valued, then we assume the following.

$$
\begin{aligned}
& g:[0, \infty] \rightarrow \boldsymbol{R}, \\
& G(z)=g(|z|) \text { for complex } z, \\
& F(z)=g^{\prime}(z) z /|z| .
\end{aligned}
$$

It follows that

$$
\left.\frac{\partial}{\partial \varepsilon} G(z+\varepsilon \zeta)\right|_{\varepsilon=0}=\operatorname{Re} F(z) \bar{\zeta}
$$

and

$$
\frac{\partial}{\partial x_{j}} G(u(x))=\operatorname{Re} F(u(x)) \frac{\partial \bar{u}}{\partial x_{j}} .
$$

In this case, the middle term in Equation (7) should be $(2-n) \operatorname{Re} \int \bar{u} F(u) d x$.

Proof of Theorem 1. We have $-(\Delta u) \bar{u}=-\nabla(\nabla u \bar{u})+|\nabla u|^{2}$. So if we multiply Equation (6) by $\bar{u}$, integrate, and assume that $u$ and its derivatives are small enough at infinity, we obtain

$$
\int\left\{|\nabla u|^{2}+\operatorname{Re} \bar{u} F(u)\right\} d x=0 .
$$

On the other hand, the multiplier $r \partial \bar{u} / \partial r=\sum_{i} x_{i} \bar{u}_{i}$, where we denote derivatives by subscripts, gives the identities:

$$
\begin{aligned}
& -\operatorname{Re} u_{j j} x_{i} \bar{u}_{i}=-\operatorname{Re}\left(u_{j} x_{i} \bar{u}_{i}\right)_{j}+\left(\frac{1}{2} x_{i}\left|u_{j}\right|^{2}\right)_{i}+\left(1-\frac{n}{2}\right)\left|u_{j}\right|^{2}, \\
& \operatorname{Re} F(u) x_{i} \bar{u}_{i}=\left(x_{i} G(u)\right)_{i}-n G(u), \\
& \int\left\{(n-2)|\nabla u|^{2}+2 n G(u)\right\} d x=0 .
\end{aligned}
$$

This proves (7). It may be illuminating to give a different proof of the second equality in (7). We regard $u$ as a solution of the variational problem $\delta \mathscr{L}(u)=0$, where

$$
\mathscr{L}(u)=\int\left\{\frac{1}{2}|\nabla u|^{2}+G(u)\right\} d x .
$$

Motivated by the fact that $r \partial / \partial r$ is the infinitesimal generator of the scale change $u(x) \rightarrow u(\lambda x)$, we define $u_{\lambda}(x)=\lambda^{(n-2) / 2} u(\lambda x)$ for $\lambda>0$. Then

$$
\mathscr{L}\left(u_{\lambda}\right)=\int\left\{\frac{1}{2} \lambda^{n}|(\nabla u)(\lambda x)|^{2}+G\left(\lambda^{(n-2) / 2} u(\lambda x)\right)\right\} d x
$$


Hence

$$
0=\left.\frac{d}{d \lambda} \mathscr{L}\left(u_{\lambda}\right)\right|_{\lambda=1}=\int\left\{-n G(u)+\frac{n-2}{2} \operatorname{Re} \bar{u} F(u)\right\} d y .
$$

Remark. The last proof is based on the transformation $u \rightarrow u_{\lambda}$, which leaves the Dirichlet integral invariant. For which functions $G$ does it also leave the integral $\int G(u) d x$ invariant? Exactly those $G$ for which $2 n G(z)=(n-2) \operatorname{Re} \bar{z} F(z)$. That is, $2 n g(s)=(n-2) s g^{\prime}(s)$, whence

$$
G(z)=-C|z|^{q+1}, \quad q=\frac{n+2}{n-2}, \quad \text { if } n>2 .
$$

Now a critical point of the functional $\mathscr{L}(u)$ is the same as a critical point of the Dirichlet integral subject to the constraint $\int|u|^{q+1} d x=1$. Let

$$
m=\inf \left\{\int|\nabla u|^{2} d x\right\}^{1 / 2} \text { subject to } \int|u|^{q+1} d x=1
$$

( $u$ a test function), then $m\|u\|_{q+1} \leqq\|\nabla u\|_{2}$. This is the classical Sobolev inequality.

We are going to prove Theorem 2 by means of the variational problem

$$
\min \int\left\{\frac{1}{2}|\nabla u|^{2}+\frac{1}{2} u^{2}+G_{1}(u)\right\} d x
$$

subject to the constraint $\int G_{2}(u) d x=1, u \in H^{1}$. The following theorem serves as motivation for our proof. We have made some simplifications; in particular, we consider the real case only.

Theorem 3. Let $F_{1}$ and $F_{2}$ be continuous functions satisfying (8) and (9) and $F_{i}(s)=O(s)$ as $s \rightarrow \infty$. Extend $F_{i}(s)$ to be zero for $s<0$. If the above problem has a solution, then it has a solution which is non-negative, radial and exponentially decaying.

Proof. Let $u$ be a solution. Let $\left.u^{+}(x)=\max (u(x)), 0\right)$. Then $G_{i}\left(u^{+}\right)=G_{i}(u), \nabla\left(u^{+}\right)$ $=(\nabla u)^{+}$and $u^{+} \in H^{1}$. So $u^{+}$is a solution of the same minimum problem and of course $u^{+}$is non-negative. In the rest of the proof we assume $u \geqq 0$. To find a radial solution we use Steiner symmetrization. In the classical use of this technique (Polya and Szegö [8], Weinberger [15]), the integrands are quadratic, the equation is linear and the domain is bounded. However, the proof easily extends to the present case. Let $D=\left\{(x, t) \in \boldsymbol{R}^{n+1}: 0 \leqq t \leqq u(x)\right\}$. Let $D^{*}$ be the symmetrization of $D$ in the hyperplane $x_{1}=0$. This means that each line $L$ in $\boldsymbol{R}^{n+1}$ perpendicular to $x_{1}=0$ intersects $D^{*}$ in a single line segment symmetric about $x_{1}=0$ such that the length of $L \cap D^{*}$ equals the length of $L \cap D . D^{*}$ is of the form $D^{*}=\left\{(x, t) \in \boldsymbol{R}^{n+1}: 0 \leqq t \leqq u^{*}(x)\right\}$. Then (for $i=1,2$ )

$$
\begin{aligned}
\int G_{i}(u(x)) d x & =\iint_{0}^{u(x)} F_{i}(t) d t d x=\iint_{D} F_{i}(t) d x d t \\
& =\iint_{D^{*}} F_{i}(t) d x d t=\iint_{0}^{u^{*}(x)} F_{i}(t) d t d x=\int G_{i}\left(u_{*}(x) d x,\right.
\end{aligned}
$$

where the middle equality follows from the definition of $D^{*}$. On the other hand, symmetrization does not increase surface area. We apply this fact to the boundary of

$$
D_{R, \varepsilon}=\left\{(x, t) \in \boldsymbol{R}^{n+1}:|x|<R, 0 \leqq t \leqq \varepsilon u(x)\right\}
$$


where $R$ and $\varepsilon$ are positive numbers. Thus

$$
\begin{aligned}
\int_{|x|<R}\left\{1+\varepsilon^{2}|\nabla u|^{2}\right\}^{1 / 2} d x & +\int_{|x|=R} \varepsilon u(x) d S \\
& \geqq \int_{|x|<R}\left\{1+\varepsilon^{2}\left|\nabla u^{*}\right|^{2}\right\}^{1 / 2} d x+\int_{|x|=R} \varepsilon u^{*}(x) d S .
\end{aligned}
$$

Since $u-u^{*} \in L^{2}$, we may choose a sequence $R_{n} \rightarrow \infty$ so that the integral of $\left|u-u^{*}\right|$ over $|x|=R_{n}$ tends to zero. Noting that $0 \leqq\left\{1+\varepsilon^{2}|\nabla u|^{2}\right\}^{1 / 2}-1 \leqq \frac{1}{2} \varepsilon^{2}|\nabla u|^{2}$, and taking $R=R_{n} \rightarrow \infty$, we obtain

$$
\left.\int\left[\left\{1+\varepsilon^{2}|\nabla u|^{2}\right\}^{1 / 2}-1\right] d x \geqq \int\left\{1+\varepsilon^{2}\left|\nabla u^{*}\right|^{2}\right\}^{1 / 2}-1\right] d x .
$$

Dividing both sides by $\varepsilon^{2}$ and letting $\varepsilon \rightarrow 0$, we get

$$
\int|\nabla u|^{2} d x \geqq \int|\nabla u *|^{2} d x \text {. }
$$

This shows that $u^{*}$ is a solution of the same minimum problem as $u$. If we repeat this procedure successively over an appropriate sequence of hyperplanes, we get a nonnegative radial solution.

Call the radial solution $u$. It is continuous for $r=|x| \neq 0$, by the Radial Lemma of Section 3. It satisfies an equation

$$
u_{r r}+\frac{n-1}{r} u_{r}-F(u)=0, \quad 0<r<\infty,
$$

where $F(u)=u+F_{1}(u)-\lambda F_{2}(u)$. Thus $r^{1-n}\left(r^{n-1} u_{r}\right)_{r}=u_{r r}+((n-1) / r) u_{r}$ is continuous and hence $u$ is a $C^{2}$ function for $r \neq 0$. Let

$$
q(r)=\frac{F(u(r))}{u(r)}=1+F_{1}^{\prime}(0)+p(r) .
$$

By (8) and (9), $p(r) \rightarrow 0$ as $r \rightarrow \infty$. Hence $q(r) \geqq \frac{1}{2}$ for large enough $r$. Now $v=r^{(n-1) / 2} u$ satisfies the equation

$$
v_{r r}-\left[q(r)+\frac{(n-1)(n-3)}{4 r^{2}}\right] v=0,
$$

whence

$$
\left(\frac{1}{2} v^{2}\right)_{r r}=v_{r}^{2}+\left[q(r)+\frac{(n-1)(n-3)}{4 r^{2}}\right] v^{2} .
$$

Thus $w=v^{2}$ satisfies the inequality $w_{r r} \geqq w$ for large enough $r$. From this inequality follows the exponential decay of $w$, hence of $u$.

In fact, it implies that $Q=e^{-r}\left(w_{r}+w\right)$ in non-decreasing for large $r$. If $Q$ remains non-positive for large $r$, then $\left(e^{r} w\right)_{r}=e^{2 r} Q \leqq 0$, which implies that $w=O\left(e^{-r}\right)$ as $r \rightarrow \infty$. If, on the other hand, $Q \geqq 2 \delta>0$, then $w_{r}+w$ is certainly not integrable near $\infty$. But the functions $v^{2}, v_{r}^{2}, w$ and $w_{r}$ are all integrable on an interval $R<r<\infty$ because $u \in H^{1}$. This contradiction proves the exponential decay.

\section{Existence}

Radial Lemma 1. Let $n \geqq 2$. Every radial function $u \in H^{1}$ is almost everywhere equal to a function $U(x)$, continuous for $x \neq 0$, such that

$$
|U(x)| \leqq c|x|^{(1-n) / 2}\|u\|_{H^{1}} \quad \text { for } \quad|x| \geqq 1,
$$

where $c$ depends only on $n$. 
Proof. Let $m=(n-1) / 2$. For $v \in C^{\infty} \cap H^{1}$, we have

$$
\begin{aligned}
\left(r^{2 m} v^{2}\right)_{r} & =\left(\left(r^{m} v\right)^{2}\right)_{r}=2\left(r^{m} v\right)_{r} r^{m} v \leqq\left(r^{m} v\right)_{r}^{2}+\left(r^{m} v\right)^{2} \\
& =r^{n-1}\left(v_{r}^{2}+v^{2}\right)+m\left(r^{n-2} v^{2}\right)_{r}-\frac{(n-1)(n-3)}{4} r^{n-3} v^{2} .
\end{aligned}
$$

If $n \geqq 3$, we integrate over an interval $[0, r]$ to obtain

$$
\begin{aligned}
r^{n-1} v^{2}(r) & \leqq \int_{0}^{r}\left(v_{r}^{2}+v^{2}\right) \varrho^{n-1} d \varrho \\
& =\left|S^{n-1}\right|^{-1}\|v\|_{H^{1}}^{2},
\end{aligned}
$$

where $\left|S^{n-1}\right|$ is the area of the unit sphere in $\boldsymbol{R}^{n}$. If $u \in H^{1}$, we let

$$
v_{\varepsilon}(r)=\int_{-\varepsilon}^{0} \zeta_{\varepsilon}(\varrho) u(r-\varrho) d \varrho,
$$

where $\zeta_{\varepsilon}$ is the usual approximate delta function, and pass to the limit to obtain the result. In case $n=2$, the differential inequality takes the form

$$
-\left(r v^{2}\right)_{r} \leqq r\left(v_{r}^{2}+v^{2}\right)+\left(\frac{1}{2} v^{2}\right)_{r}+\frac{1}{4 r} v^{2} .
$$

We integrate over an interval $[r, \infty]$ to obtain

$$
\left(r+\frac{1}{2}\right) v^{2}(r) \leqq \int_{r}^{\infty}\left(v_{r}^{2}+v^{2}\right) \varrho d \varrho+\int_{r}^{\infty} \frac{1}{4 \varrho} v^{2} d \varrho .
$$

For $r \geqq 1$, we may replace $1 / \varrho$ by $\varrho$ to obtain

$$
2 \pi\left(r+\frac{1}{2}\right) v^{2}(r) \leqq \frac{5}{4}\|v\|_{H^{1}}^{2} .
$$

The proof is completed as before.

Compactness Lemma 2. Let $\left\{P_{N}\right\}$ and $\left\{Q_{N}\right\}$ be two sequences of continuous functions: $\boldsymbol{R} \rightarrow \boldsymbol{R}$. For $c>0$, let $\gamma(c)=\sup \left\{|t|: t=P_{N}(s)\right.$ for some $N$ and such that $\left.\left|Q_{N}(s)\right| \leqq c\left|P_{N}(s)\right|\right\}$.

Assume i) $\gamma(c)<\infty$ for all $c>0$. (In other words, $P_{N} / Q_{N} \rightarrow 0$ uniformly as $P_{N} \rightarrow \infty$.)

ii) $\left\{u_{N}\right\}$ is a sequence of measurable functions: $\boldsymbol{R}^{n} \rightarrow \boldsymbol{R}$ such that

$$
q=\sup _{N} \int\left|Q_{N}\left(u_{N}(x)\right)\right| d x<\infty .
$$

iii) $P_{N}\left(u_{N}(x)\right) \rightarrow v(x)$ for a.e. $\quad x \in \boldsymbol{R}^{n}$.

a) Then

$$
\int_{B}\left|P_{N}\left(u_{N}\right)-v\right| d x \rightarrow 0
$$

for all bounded sets $B$.

b) Assume in addition that

iv) $P_{N}(s)=o\left(Q_{N}(s)\right)$ as $s \rightarrow 0$ uniformly in $N$.

v) $u_{N}(x) \rightarrow 0$ as $|x| \rightarrow \infty$, uniformly in $x$ and $N$.

Then $\int\left|P_{N}\left(u_{N}\right)-v\right| d x \rightarrow 0$. 
Proof. We use the method of [12]. Fix $N$ and $\varepsilon>0$. Let $c>4 q / \varepsilon$. Let $B$ be a bounded set. By iii) and Egorov's lemma, choose a set $Z \subset B$ of measure less than $\varepsilon /(4 \gamma(c))$ such that $P_{N}\left(u_{N}\right) \rightarrow v$ uniformly on $B / Z$. Let

$$
Z^{\prime}=\left\{x \in Z:\left|Q_{N}\left(u_{N}(x)\right)\right| \leqq c\left|P_{N}\left(u_{N}(x)\right)\right|\right\}, \quad Z^{\prime \prime}=Z \backslash Z^{\prime} .
$$

Thus $\left|P_{N}\left(u_{N}\right)\right| \leqq \gamma(c)$ on $Z^{\prime}$ and $\left|P_{N}\left(u_{N}\right)\right| \leqq c^{-1}\left|Q_{N}\left(u_{N}\right)\right|$ on $Z^{\prime \prime}$. Hence

$$
\begin{aligned}
& \int_{Z}\left|P_{N}\left(u_{N}\right)\right| d x \leqq q / c+\gamma(c)\left|Z^{\prime \prime}\right|<\varepsilon / 2, \\
& \varlimsup \varlimsup_{B}\left|P_{N}\left(u_{N}\right)-v\right| d x \leqq \varepsilon+\lim \int_{B \backslash Z}\left|P_{N}\left(u_{N}\right)-v\right| d x=\varepsilon .
\end{aligned}
$$

This proves a). To prove b), let $\varepsilon>0$. By iv) choose $\delta>0$ so that $\sup _{N}\left|P_{N}(s) / Q_{N}(s)\right| \leqq \varepsilon / 2 q$ for $|s| \leqq \delta$. By v) choose $R>0$ so that $\sup _{N}\left|u_{N}(x)\right|<\delta$ for $|x|>R$. Then

$$
\int_{|x|>R}\left|P_{N}\left(u_{N}(x)\right)\right| d x \leqq \frac{\varepsilon}{2 q} \int\left|Q_{N}\left(u_{N}(x)\right)\right| d x \leqq \frac{\varepsilon}{2} .
$$

Applying Part a) of this lemma to $B=\{|x| \leqq R\}$, we conclude that

$$
\varlimsup \lim \int\left|P_{N}\left(u_{N}\right)-v\right| d x \leqq \varepsilon \text {. }
$$

This completes the proof.

We are now ready to begin the proof of Theorem 2 . Let $F_{1}$ and $F_{2}$ satisfy conditions (8)-(11), where

$$
G_{i}(s)=\int_{0}^{s} F_{i}(t) d t
$$

We extend them by zero for negative $s: F_{i}(s)=0$ for $s<0$. We may also assume $L=-\Delta+I$.

Lemma 3. There exists a non-trivial solution of (12) in case $F_{i}(s) \leqq c_{i} s$ for all $s$ $(i=1,2)$.

Proof. In this case $0 \leqq G_{i}(s) \leqq \frac{1}{2} c_{i} s^{2}$. Let

$$
M=\inf \int\left\{\frac{1}{2}\left(|\nabla u|^{2}+u^{2}\right)+G_{1}(u)\right\} d x=\inf \mathscr{L}(u)
$$

subject to the conditions

$$
u \in H^{1}, \quad u \text { radial, } \quad \mathscr{M}(u)=\int G_{2}(u) d x=1 .
$$

By (8), $G_{2}(s)>0$ for $s>0$. So for any $\gamma>0$, there exists $u_{o} \in C_{c}^{\infty}, u_{o}$ radial, with $\mathscr{M}\left(u_{o}\right)=\gamma$. Thus $M$ is finite. Choose any minimizing sequence: $u_{v} \in H^{1}, u_{v}$ radial, $\mathscr{M}\left(u_{v}\right)=1, \mathscr{L}\left(u_{v}\right) \rightarrow M$. Then $\left\{u_{v}\right\}$ is bounded in $H^{1}$ and $\left\{G_{1}\left(u_{v}\right)\right\}$ is bounded in $L^{1}$. By Sobolev's inequality, $\left\{u_{v}\right\}$ is also bounded in $L^{l+1}$, where $l+1=2 n /(n-2)$ and $n \geqq 3$. By compactness on bounded subsets of $\boldsymbol{R}^{n}$, there is a subsequence, which we still denote by $\left\{u_{v}\right\}$, converging to a limit $u$ weakly in $H^{1}$ and almost everywhere. Hence $G_{i}\left(u_{v}\right) \rightarrow G_{i}(u)$ a.e. $(i=1,2)$. We shall apply the Compactness Lemma with $u_{N}=u_{v}$, $P_{N}=G_{2}$, and $Q_{N}(s)=|s|^{l+1}+G_{1}(s)+s^{2}$. Hypothesis i) is satisfied because $|s|^{l+1}+G_{1}(s) \leqq c G_{2}(s) \Rightarrow|s|$ bounded $\Rightarrow G_{2}(s)$ bounded. 
Hypothesis v) is satisfied because of the Radial Lemma. Thus $\mathscr{M}\left(u_{v}\right) \rightarrow \mathscr{M}(u)$. Hence $\mathscr{M}(u)=1$ and also $u$ is radial.

By Fatou's Lemma and weak convergence, we have $\mathscr{L}(u) \leqq \underline{\lim } \mathscr{L}\left(u_{v}\right) \leqq M$. By definition of $M, \mathscr{L}(u)=M$. Thus $u$ attains the minimum. Now, because $F_{1}$ and $F_{2}$ are sublinear and continuous, it is easy to check that $\mathscr{L}$ and $\mathscr{M}$ are $C^{1}$ functionals on $H_{r}^{1}=\left\{w \in H^{1}: w\right.$ is radial $\}$. Their Frechet derivatives are $\mathscr{M}^{\prime}(w) v=\int F_{2}(w) v d x$ and $\mathscr{L}^{\prime}(w) v=\int\left\{\nabla w \cdot \nabla v+w v+F_{1}(w) v\right\} d x$. Thus there exists $\lambda \in \boldsymbol{R}, u \in H^{1}$ such that $\mathscr{L}^{\prime}(u) v$ $=\lambda \mathscr{M}^{\prime}(u) v$ for all $v \in H_{r}^{1}$. Let $h=-\Delta u+u+F_{1}(u)-\lambda F_{2}(u)$. Then $h$ is radial and is orthogonal to all radial test functions. Hence $h=0$.

Remark. If the space $H_{r}^{1} \cap L^{p}$ were used, the preceding method could be made to work for functions $F_{i}$ of polynomial growth at infinity; the $F_{i}$ could have faster growth if Orlicz spaces were used. Instead, in the following argument we allow more general conditions on $F_{i}$ by using an extra passage to the limit.

Lemma 4. Let $F_{i}(s)$ be as in Theorem 2 (zero for $s<0$ ). Then

$$
G_{2}(s)=o\left(s^{l+1}+G_{1}(s)\right) \text { as } s \rightarrow \infty .
$$

For $N>0$ define the truncated functions

$$
F_{i N}(s)=\left\{\begin{array}{lll}
F_{i}(s) & \text { for } & s \leqq N \\
F_{i}(N) & \text { for } & s>N
\end{array}\right.
$$

and let $G_{i N}(s)$ be their indefinite integrals. Then

$$
s F_{2 N}(s)=O\left(s^{l+1}+G_{1 N}(s)\right) \text { as } s \rightarrow \infty \text { uniformly in } N \text {. }
$$

For any $c>0$, there exists $\gamma(c)>o$ such that

$$
s^{l+1}+G_{1 N}(s) \leqq c G_{2 N}(s) \Rightarrow G_{2 N}(s) \leqq \gamma(c) .
$$

For any $\sigma>0$, let $c_{\sigma}=\sup _{s \leqq \sigma} F_{1}(s)$; then

$$
F_{1 N}(s) \leqq c_{\sigma}+\frac{1}{\sigma} s F_{1 N}(s) \quad \text { for } \quad N>\sigma, \quad s \in \boldsymbol{R}
$$

Proof. To prove (33), we let $\sigma<N$ and consider 3 cases. For $s \leqq \sigma$, we have $F_{1 N}(s)$ $=F_{1}(s) \leqq c_{\sigma}$. For $\sigma \leqq s \leqq N$, we have $F_{1 N}(s) \leqq s F_{1}(s) / \sigma=s F_{1 N}(s) / \sigma$. For $N \leqq s$, we have $F_{1 N}(s)=F_{1}(N) \leqq s F_{1}(N) / \sigma=s F_{1 N}(s) / \sigma$. have

We also prove (31) in 3 cases. For $s \leqq N,(31)$ is identical with (11). For $s>N$, we

$$
G_{i N}(s)=\int_{0}^{s} F_{i N}=G_{i}(N)+(s-N) F_{i}(N)
$$

For $N<s<2 N$, we have from (11) and (34)

$$
F_{2}(N)=O\left(N^{l}+G_{1}(N) / N\right)=O\left(s^{l}+2 G_{1}(N) / s\right)=O\left(s^{l}+G_{1 N}(s) / s\right) .
$$

For $s \geqq 2 N$, we have from (10) and (34),

$$
F_{2}(N)=o\left(N^{l}+F_{1}(N)\right)=o\left(s^{l}+2 \frac{s-N}{s} F_{1}(N)\right)=O\left(s^{l}+G_{1 N}(s) / s\right) .
$$


To prove (30), let $\varepsilon>0$. By (10) there exists $T=T(\varepsilon)$ so large that

$$
F_{2}(t)<\varepsilon\left(t^{l}+F_{1}(t)\right) \text { for } t \geqq T \text {. }
$$

For $s \geqq T$, we integrate (35) over the interval $[T, s]$ to get

$$
G_{2}(s) \leqq \varepsilon\left(\frac{s^{l+1}}{l+1}+G_{1}(s)\right)+G_{2}(T)-\varepsilon G_{1}(T)-\varepsilon \frac{T^{l+1}}{l+1} .
$$

Thus (30) holds. That is, there exists $T^{\prime}=T^{\prime}(\varepsilon) \geqq T$ so that

$$
G_{2}(t)<\varepsilon\left(t^{l+1}+G_{1}(t)\right) \text { for } t \geqq T^{\prime} .
$$

To prove (32), let $c>0$. Let $\varepsilon=1 /(2 c)$. Let $s$ satisfy the inequality

$$
s^{l+1}+G_{1 N}(s) \leqq c G_{2 N}(s) \text {. }
$$

Case 1: $s \leqq N$. Then $G_{i N}(s)=G_{i}(s)$, so that (36) and (37) imply that $s \leqq T^{\prime}$. Hence $G_{2 N}(s) \leqq G_{2}\left(T^{\prime}\right)=\gamma(c)$.

Case 2: $s>N$. By (34), (37) takes the form

$$
s^{l+1}+G_{1}(N)+(s-N) F_{1}(N) \leqq c\left\{G_{2}(N)+(s-N) F_{2}(N)\right\} .
$$

In this case, we will show that $N<T^{\prime}=T^{\prime}(1 /(2 c))$. Thus $N$ runs through a finite number of integers. By (37), $s$ is also bounded by a function of $c$. Hence so is $G_{2 N}(s)$. It remains to prove a contradiction if we suppose that $N \geqq T^{\prime}$. In that case, we may choose $t=N$ in both (35) and (36). Then we add (s-N) times (35) to (36) to obtain

$$
G_{2}(N)+(s-N) F_{2}(N)<\frac{1}{2 c}\left\{N^{l+1}+(s-N) N^{l}+G_{1}(N)+(s-N) F_{1}(N)\right\} .
$$

This indeed contradicts $\left(37^{\prime}\right)$.

Proof of Theorem 2. For fixed $N$, we apply Lemma 3. There exists $u_{N} \in H^{1}, u_{N}$ radial, $u_{n}$ minimizes

$$
\left.\mathscr{L}_{N}(v)=\int \frac{1}{2}\left(|\nabla v|^{2}+v^{2}\right)+G_{1 N}(v)\right\} d x
$$

subject to the constraints that $v \in H^{1}, v$ is radial and $G_{2 N}(v) d x=1$. Furthermore,

$$
-\Delta u_{N}+u_{N}+F_{1 N}\left(u_{N}\right)=\lambda_{N} F_{2 N}\left(u_{N}\right) .
$$

Multiplying (38) by $u_{N}$ and integrating, we find

$$
\int\left\{\left|\nabla u_{N}\right|^{2}+u_{N}^{2}+u_{N} F_{1 N}\left(u_{N}\right)\right\} d x=\lambda_{N} \int u_{N} F_{2 N}\left(u_{N}\right) d x .
$$

Now choose $u_{o}$ as in the proof of Lemma 3. Let $N>\sup \left|u_{o}(x)\right|$. Then $G_{i N}\left(u_{o}\right)=G_{i}\left(u_{o}\right)$. By the minimization property of $u_{N}$, we have

$$
\mathscr{L}_{N}\left(u_{N}\right) \leqq \mathscr{L}_{N}\left(u_{o}\right)=\mathscr{L}\left(u_{o}\right)<\infty .
$$

Thus $\left\{u_{N}\right\}$ is bounded in $H^{1}$ and $\int G_{1 N}\left(u_{N}\right) d x$ is bounded. By Sobolev's inequality, $\left\{u_{N}\right\}$ is bounded in $L^{l+1}$. By $(31), \int u_{N} F_{2 N}\left(u_{N}\right) d x$ is also bounded.

By compactness, there is a subsequence, still denoted by $\left\{u_{N}\right\}$, such that $u_{N}$ converges to some limit $u$ weakly in $H^{1}$ and a.e. Hence $G_{i N}\left(u_{N}\right) \rightarrow G_{i}(u)$ and $F_{i N}\left(u_{N}\right) \rightarrow F_{i}(u)$ a.e. We now apply the Compactness Lemma $2 \mathrm{~b}$ ) with

$$
P_{N}(s)=G_{2 N}(s) \text { and } Q_{N}(s)=|s|^{l+1}+G_{1 N}(s)+s^{2} .
$$


Assumption i) of Lemma 2 is identical with (32), while Assumption v) follows from the Radial Lemma 1. Therefore $\int G_{2}(u) d x=1$.

Lemma 5. $\left\{\lambda_{N}\right\}$ is a bounded sequence of positive numbers.

Assuming the validity of Lemma 5, the right side of (39) is bounded, whence $\int u_{N} F_{1 N}\left(u_{N}\right) d x$ is also bounded. By the Compactness Lemma $\left.2 \mathrm{a}\right)$ with $P_{N}=F_{i N}$ and $Q_{N}(s)=s F_{i N}(s)$, we deduce that $F_{i N}\left(u_{N}\right) \rightarrow F_{i}(u)$ in $L_{\text {loc }}^{1}$ for $i=1,2$. Taking a subsequence $\lambda_{N} \rightarrow \lambda$, we see that each term in (38) converges in the sense of distributions. We conclude that

$$
-\Delta u+u+F_{1}(u)=\lambda F_{2}(u) \text {. }
$$

To show $u$ is non-negative, let $u^{-}(x)=\min (u(x), 0)$ and let $A=\{x: u(x)<0\}$. Since $u \in H^{1}$, it follows that $u^{-} \in H^{1}$ and $\partial\left(u^{-}\right) / \partial x_{j}=\left(\partial u / \partial x_{j}\right)^{-}$. Multiplying the differential equation by $u^{-}$, we obtain

$$
\begin{aligned}
0 & =\int\left\{\nabla u \cdot \nabla u^{-}+u u^{-}+F(u) u^{-}\right\} d x \\
& =\int_{A}\left\{|\nabla u|^{2}+|u|^{2}\right\} d x .
\end{aligned}
$$

Hence $u=0$ on $A$ so that $u \geqq 0$. (Another proof could be based on the method of Theorem 3.) Finally, by the proof of Theorem $3, u$ is a $C^{2}$ function for $x \neq 0$ which decays exponentially as $r \rightarrow \infty$. We also note that from the differential equation we have

$$
\int\left\{|\nabla u|^{2}+u^{2}+u F_{1}(u)\right\} d x=\lambda \int u F_{2}(u) d x,
$$

from which it follows that $\lambda \geqq 0$. If $\lambda=0$, then $u=0$ and $\int G_{2}(u) d x=0$, which is a contradiction.

Proof of Lemma 5. It follows from (39) that $\lambda_{N}>0$. Now fix $v \in C_{c}^{\infty}$. By (38),

$$
\lambda_{N}\left(F_{2 N}\left(u_{N}\right), v\right)=\left(\nabla u_{N}, \nabla v\right)+\left(u_{N}, v\right)+\left(F_{1 N}\left(u_{N}\right), v\right) .
$$

By (33),

$$
\begin{aligned}
\lambda_{N}\left|\left(F_{2 N}\left(u_{N}\right), v\right)\right| & \leqq\left\|u_{N}\right\|_{H^{1}}\|v\|_{H^{1}}+c_{\sigma}\|v\|_{L^{1}}+\sigma^{-1}\|v\|_{L^{\infty}}\left(u_{N}, F_{1 N}\left(u_{N}\right)\right) \\
& \leqq k\left(1+c_{\sigma}+\sigma^{-1} \lambda_{N}\right)
\end{aligned}
$$

with $k$ independent of $\sigma$ and $N$, where we have used (39) and the known bounds. Given $\varepsilon>0$, choose $N>\sigma=k / \varepsilon$. Then

$$
\left|\left(F_{2 N}\left(u_{N}\right), v\right)\right| \leqq \lambda_{N}^{-1} k\left(1+c_{\sigma}\right)+\varepsilon .
$$

If $\left\{\lambda_{N}\right\}$ were unbounded, then a subsequence would satisfy $\lambda_{N} \rightarrow \infty$ and so $\left(F_{2 N}\left(u_{N}\right), v\right) \rightarrow 0$. On the other hand, by the Compactness Lemma $\left.2 a\right)$ just as above, $\left(F_{2 N}\left(u_{N}\right), v\right) \rightarrow\left(F_{2}(u), v\right)$. Thus $\left(F_{2}(u), v\right)=0$ for all test functions $v \in C_{c}^{\infty}$. Hence $F_{2}(u)=0$. By $(8), G_{2}(u)=0$. This contradicts $\int G_{2}(u) d x=1$ and completes the proof of Lemma 5 and Theorem 2.

Remark. If the dimension $n=1$ or 2 , Theorem 2 is valid under weaker conditions. If $n=2$, we replace $(n+2) /(n-2)$ by an arbitrary positive number. Since $H^{1}$ is contained in a certain exponential Orlicz space $L^{\Phi}$, we actually need only replace 
$s^{l+1}$ by $\Phi(s)$. The proof is unchanged. If $n=1$, strongly convergent sequences in $H^{1}$ converge uniformly; hence the direct method of Lemma 3 works. Theorem 2 is valid without any growth conditions (10), (11).

Remark. Our proof shows that, for each $\gamma>0$, there is a solution with $\int G_{2}(u) d x=\gamma$.

Remark. There exists a non-positive as well as a non-negative solution if $F_{i}$ are continuous real functions satisfying

$$
s F_{1}(s) \geqq 0, \quad s F_{2}(s)>0
$$

and (9), (10), (11) with $s^{l}$ replaced by $|s|^{l}$, and $\infty$ by $\pm \infty$.

\section{Existence of Many Solutions}

Theorem 4. Let $L=I-\Delta$. Let $F_{1}=0$. Let $F_{2}$ be a continuous real function such that

$$
\begin{aligned}
& s F_{2}(s)>0 \text { for } s \neq 0, \\
& F_{2}(s)=O\left(|s|^{p}\right) \text { as }|s| \rightarrow \infty \text { where } p<(n+2) /(n-2)=l, \\
& F_{2} \text { is odd and } F_{2}(0)=0 .
\end{aligned}
$$

For any $\gamma>0$, there exists an infinite sequence of distinct pairs of radial solutions of (12),

$$
\left(\lambda_{k}, \pm u_{k}\right) \quad k=0,1,2, \ldots,
$$

with $\left(L u_{k}, u_{k}\right)=\gamma$.

Proof. We apply Theorem 2.10 of Rabinowitz [10], which is based on the minimax characterization of the higher eigenvalues. We use the space $E=H_{r}^{1}$ (the radial functions in $H^{1}$ ) and the functional $f(u)=\int G_{2}(u) d x$. We need only verify the hypothesis of Lemma 2.11 of [10]. Let $u_{N} \rightarrow u$ weakly in $H_{r}^{1}$, hence in $L^{l+1}$. By the Compactness and Radial Lemmas and (42), we have $F_{2}\left(u_{N}\right) \rightarrow F_{2}(u)$ strongly in $L^{p^{\prime}}$, where $\left(p^{\prime}\right)^{-1}+p^{-1}=1$, and hence strongly in $\left(H_{r}^{1}\right)^{*}$.

A special case of Theorem 4 can be found in Berger [2]. The following theorem is based on a variational method without constraints.

Theorem 5. Let $F$ be a real continuous function which satisfies (42), as well as

$$
\begin{aligned}
& F(s) / s \rightarrow-\infty \text { as } \quad s \rightarrow+\infty, \\
& s F(s) \geqq \alpha G(s) \text { for some } \quad \alpha>2, \\
& F(s)=o(s) \text { as } \quad s \rightarrow 0 .
\end{aligned}
$$

Then the equation $L u+F(u)=0$ possesses at least one non-trivial solution. If $F$ is odd, there is an infinite number of distinct pairs of solutions, $\pm u_{k}(k=0,1,2, \ldots)$.

Proof. We apply Theorems 3.9 and 3.37 of [10]. We use $L=I-\Delta, E=H_{r}^{1}$ and

$$
f(u)=\int\left\{\frac{1}{2}\left(|\nabla u|^{2}+u^{2}\right)+G(u)\right\} d x .
$$


The only novel feature is the verification of condition (PS) ${ }^{+}$of $[10]$. Let $\left\{u_{N}\right\}$ be a sequence satisfying

$$
\begin{aligned}
& f\left(u_{N}\right) \leqq d, \\
& h_{N}=-\Delta u_{N}+u_{N}+F\left(u_{N}\right) \rightarrow 0 \text { in } H^{-1} .
\end{aligned}
$$

Just as in [10], p. 172, these conditions imply that $\left\{u_{N}\right\}$ is bounded in $H_{r}^{1}$. As in the proof of Theorem $4, F\left(u_{N}\right) \rightarrow F(u)$ strongly in $L^{p^{\prime}}$ and $h_{N} \rightarrow 0$ strongly in $H^{-1}$, for a subsequence which converges weakly to some $u$ in $H^{1}$ and a.e. Hence

$$
\left\|u_{N}-u\right\|_{H^{1}}^{2}=-\left(F\left(u_{N}\right)-F(u), u_{N}-u\right)+\left(h_{N}, u_{N}-u\right) \rightarrow 0 \text {. }
$$

This proves (PS) ${ }^{+}$and Theorem 5 .

Example 1. $-\Delta u+u-|u|^{q-1} u=0$, where $1<q<(n+2) /(n-2)$. Both Theorems 4 and 5 imply the existence of an infinite sequence of radial solutions $u_{0}, u_{1}, u_{2}, \ldots$ (If we apply Theorem 4 , the eigenvalue $\lambda_{k}$ has to be scaled out.) The solution $u_{k}$ has exactly $k$ nodes as a function of $r$. For some computer-generated solutions, see [1]. Example 2. $-\Delta u+u+|u|^{p-1} u-\lambda \mid u^{q-1} u=0$, where $1<p<q<(n+2) /(n-2)$. Theorem 5 is applicable for every $\lambda>0$. The case of larger $p$ and $q$ is open.

\section{References}

1. Anderson,D., Derrick,G.: Stability of time-dependent particlelike solutions in nonlinear field theories. J. Math. Phys. 11, 1336-1346 (1970); 12, 945-952 (1971)

2. Berger, M.S.: On the existence and structure of stationary states for a nonlinear Klein-Gordon equation. J. Funct. Anal. 9, 249-261 (1972)

3. Clément,P.: Positive eigenfunctions for a class of second-order elliptic equations with strong nonlinearity (preprint)

4. Dancer,E. N.: Boundary value problems for ordinary differential equations in infinite intervals. Proc. London Math. Soc. 30, 76-94 (1975)

5. Kato, T.: Growth properties of solutions of the reduced wave equation with a variable coefficient. Comm. Pure Appl. Math. 12, 403-425 (1959)

6. Nehari,Z.: On a nonlinear differential equation arising in nuclear physics. Proc. Roy. Irish Acad. 62, 117-135 (1963)

7. Pohozaev,S.I. : Eigenfunctions of the equation $\Delta u+\lambda f(u)=0$. Sov. Math. Doklady 5, 1408-1411 (1965)

8. Polya,G., Szegö,G.: Isoperimetric inequalities in mathematical physics. Princeton: University Press 1951

9. Rabinowitz,P.H.: Some aspects of nonlinear eigenvalue problems. Rocky Mtn. J. Math. 3, 161-202 (1973)

10. Rabinowitz,P.H.: Variational methods for nonlinear eigenvalue problems. In : Eigenvalues of nonlinear problems. Rome: C.I.M.E., Edizioni Cremonese 1974

11. Rabinowitz,P.H.: Variational methods for nonlinear elliptic eigenvalue problems. Indiana U. Math. J. 23, $729-754$ (1974)

12. Strauss, W. A. : On weak solutions of semi-linear hyperbolic equations. Anais Acad. Brasil. Ciencias 42, 645-651 (1970)

13. Stuart, C. A.: Battelle Inst. math. report No. 75,1973

14. Synge,J.L.: On a certain non-linear differential equation. Proc. Roy. Irish Acad. 62, 17-41 (1961)

15. Weinberger,H.: Variational methods for eigenvalue approximation. Philadelphia: SIAM 1974

Communicated by A. Jaffe 